\title{
INSTRUMENTOS PARA AVALIAÇÃO DA LINGUAGEM PÓS-LESÃO CEREBROVASCULAR ESQUERDA
}

\section{Instruments for language assessment following a left brain damage}

\author{
Karina Carlesso Pagliarin (1), Camila Rosa de Oliveira (2), Bruna Matias da Silva ${ }^{(3)}$, \\ Luara de Freitas Calvette ${ }^{(4)}$, Rochele Paz Fonseca ${ }^{(5)}$
}

\section{RESUMO}

O tema deste estudo é a avaliação padronizada da linguagem em pacientes com lesão cerebrovascular esquerda (LHE) que pode ser muito útil no estabelecimento do diagnóstico, prognóstico e plano terapêutico, complementando observação, entrevistas e tarefas clínicas. Este estudo teve como objetivo identificar quais instrumentos de investigação de linguagem têm sido utilizados para avaliação de quadros neurológicos súbitos envolvendo o hemisfério esquerdo (HE) e analisar quais componentes linguísticos são os mais avaliados. Foi possível identificar nove instrumentos utilizados internacionalmente que avaliam diferentes componentes linguísticos em pacientes com LHE; no contexto nacional, porém, foram encontradas apenas duas baterias para avaliação dessa população, ficando evidente a necessidade da construção e/ou de adaptação de instrumentos para a população brasileira. Os componentes linguísticos mais investigados foram nomeação e compreensão oral, respectivamente. A avaliação da linguagem torna-se essencial nos quadros envolvendo o HE, pois déficits linguísticos podem acarretar prejuízos sociais, ocupacionais e familiares.

DESCRITORES: Avaliação; Acidente Vascular Cerebral; Testes de Linguagem

\section{INTRODUÇÃO}

Esta revisão sistemática aborda um panorama da literatura nacional e internacional acerca dos

(1) Fonoaudióloga; Doutoranda em Psicologia pela Pontifícia Universidade Católica do Rio Grande do Sul, PUCRS, Porto Alegre, RS, Brasil; Bolsista CNPq.

(2) Psicóloga; Mestranda em Psicologia pela Pontifícia Universidade Católica do Rio Grande do Sul PUCRS, Porto Alegre, RS, Brasil; Bolsista CNPq.

(3) Acadêmica do Curso de Psicologia da Universidade do Vale do Rio dos Sinos, UNISINOS, São Leopoldo, RS, Brasil; Bolsista CNPq.

(4) Psicóloga; Mestranda em Psicologia pela Pontifícia Universidade Católica do Rio Grande do Sul, PUCRS, Porto Alegre, RS, Brasil; Bolsista Capes.

(5) Psicóloga e Fonoaudióloga; Professora da Faculdade de Psicologia e o Programa de Pós-graduação em Psicologia, área de concentração Cognição Humana, da Pontifícia Universidade Católica do Rio Grande do Sul PUCRS, Porto Alegre, RS, Brasil; Doutora em Psicologia pela Universidade Federal do Rio Grande do Sul; Pós-Doutora em Ciências Biomédicas, Centro de Neuroimagem pela Universidade de Montreal e em Neurorradiologia pela Faculdade de Medicina, UFRJ.

Fonte de auxílio à pesquisa: CNPq e FAPERGS

Conflito de interesses: inexistente instrumentos de exame linguístico utilizados pós-lesão cerebral de hemisfério esquerdo (HE). Em termos históricos, o HE e as funções linguísticas a ele relacionadas têm sido foco de diversos estudos, sendo por muitos anos considerado dominante para a linguagem para a maioria da população destra ${ }^{1,2}$. Desta forma, o HE apresenta um importante papel no processamento de aspectos estruturais e formais da linguagem, como morfológicos, fonológicos, sintáticos e semânticos que, quando alterados, podem gerar prejuízos comunicacionais significantes, gerando quadros afásicos ${ }^{3}$.

As alterações mais frequentes decorrentes de lesão cerebral esquerda (LHE) podem envolver problemas articulatórios, agramatismos, anomias, dificuldades compreensivas, discalculia, apraxias, prejuízos sintáticos e/ou lexicais ${ }^{3-6}$. A avaliação, nesses casos, deve abordar diferentes níveis e componentes linguísticos, dentre eles compreensão e expressão, tanto oral como escrita, considerando-se, ainda, forma, conteúdo e uso da linguagem, nos níveis da palavra, sentença e discurso. Este processo avaliativo é essencial para a identificação de estratégias para uma reabilitação eficaz ${ }^{7-9}$. 
Dentre os testes de avaliação de linguagem existentes, destaca-se o Aphasia Diagnostic Profiles ${ }^{10}$, Mississippi Aphasia Screening Test (MAST) ${ }^{11}$, Boston Assessment of Severe Aphasia (BASA) ${ }^{12}$, ASHA FACS ${ }^{13}$, Multilingual Aphasia Examination (MAE) ${ }^{14}$, Porch Index Communicative Ability (PICA) ${ }^{15}$, Frenchay Aphasia Screening Test (FAST) ${ }^{16}$, Western Aphasia Battery ${ }^{17}$ e 0 Boston Diagnostic Aphasia Examination (BDAE) ${ }^{18}$, sendo que este último é um dos instrumentos mais utilizado internacionalmente para detecção de afasia, abrangendo diversos componentes linguísticos, e se encontra adaptado e normatizado para a população brasileira ${ }^{19,20}$, estando, no entanto, disponível apenas para finalidade de pesquisa.

Embora as alterações pós LHE serem mais estudadas e frequentemente encaminhadas para a clínica fonoaudiológica de afasiologia, não há instrumentos padronizados disponíveis para o exame de afasias em nível nacional. Em contrapartida, apesar de ainda pouco abordado, principalmente em estudos nacionais, o hemisfério direito (HD), fundamental para a comunicação, principalmente em relação ao discurso, a pragmática, a prosódia e ao processamento léxico-semântico ${ }^{21-23}$ já existe instrumento específico devidamente adaptado e padronizado para a população brasileira para a avaliação deste hemisfério após acidente vascular cerebral (AVC) ou outros quadros neurológicos ou psiquiátricos que acometam a estrutura ou a função comunicativa da metade direita do cérebro ${ }^{24}$. Entretanto, estudos têm sido realizados com intuito de analisar o desempenho de indivíduos saudáveis com o teste $\mathrm{BDAE}^{20,25}$ e a influencia da idade e escolaridade com o protocolo MT Beta-86 Modificado da Bateria Montreal-Toulouse ${ }^{26-28}$ na população brasileira.

Percebe-se, assim, uma grande demanda de testes adaptados para o Português Brasileiro, tendo-se em vista que os instrumentos utilizados na clínica são meramente traduções de ferramentas de outras línguas e, muitas vezes, não condizem com questões culturais e sócio-demográficas da população em questão ${ }^{19,29}$, podendo, dessa forma, dificultar o diagnóstico, reabilitação e prognóstico desses pacientes.

Devido à ainda restrita disponibilidade de instrumentos de avaliação comportamental da linguagem elaborados e/ou adaptados para o Português Brasileiro, este estudo buscou, por meio de uma revisão sistemática, identificar quais instrumentos de investigação de linguagem têm sido utilizados para avaliar populações clínicas de adultos com quadros neurológicos súbitos envolvendo o $\mathrm{HE}$, bem como analisar quais componentes linguísticos são mais avaliados.

\section{MÉTODO}

Realizou-se uma busca na base de dados PubMed e LILACS, incluindo abstracts de artigos publicados nos últimos 10 anos (de 2001 a 2011), sendo ou não de periódicos de acesso livre. Foram utilizadas as seguintes associações de palavras-chave para a busca na base de dados PubMed: construto de avaliação AND construto de componentes linguísticos $A N D$ construto de população examinada. Para cada construto, as palavras-chave utilizadas foram a) avaliação: "evaluation" $O R$ "assessment" OR "test" OR "instrument"; b) componentes linguísticos: "phonology" OR "discourse" $O R$ "pragmatic" OR"semantic" OR"prosody" OR"writing" OR "syntax"; OR "language comprehension" OR "reading" OR "texf" OR "spontaneous speech" OR "repetition" OR "naming" OR "verbal fluency" $O R$ "language production" OR "lexical". Destaca-se que as palavras-chave deste construto, foram selecionadas com base nas tarefas que compõem instrumentos de avaliação de linguagem, consultados em compêndios neuropsicológicos; c) população examinada: "stroke" OR "aphasia" OR "left hemisphere damage". Por fim, as buscas foram feitas com limites de língua (inglês, francês, espanhol e português) e de faixa etária (acima de 19 anos). $\mathrm{Na}$ base de dados LILACS foram utilizados os mesmos construtos da pesquisa do PubMed porém, com as palavras-chave em português: "avaliação" $O R$ "teste" OR "instrumento" AND "acidente vascular cerebral" OR "afasia" $A N D$ "fonologia" OR "discurso" $O R$ "pragmática" $O R$ "escrita" $O R$ "semântica" $O R$ "sintaxe" OR "compreensão" OR "leitura" OR "texto" $O R$ "repetição" $O R$ "nomeação" $O R$ "fluência" $O R$ "fala" OR "léxico".

A partir dessas buscas, foram encontrados 310 resumos, sendo 293 no PubMed e 17 no LILACS, dos quais foram selecionados para a análise posterior de textos completos os que atenderam aos critérios: a) ser um estudo empírico b) utilizar baterias completas, breves ou escalas funcionais de avaliação da linguagem, c) incluir pacientes com LHE e d) apresentar no mínimo um instrumento para avaliação da linguagem publicado e padronizado para a população pesquisada. Foram excluídos artigos a) que se repetiam nas bases de dados, b) que utilizavam apenas escalas ou screenings que avaliavam outras funções além da linguagem, por exemplo o Mini Exame do Estado Mental, c) aqueles que não explicitavam precisamente os instrumentos de avaliação da linguagem utilizados no estudo e d) aqueles em que não havia nenhum caso de LHE súbita na população estudada. Desses, 46 artigos completos foram selecionados, sendo os instrumentos utilizados nos estudos apresentados nessa revisão. O fluxo de seleção de artigos pode ser visualizado na Figura 1. 


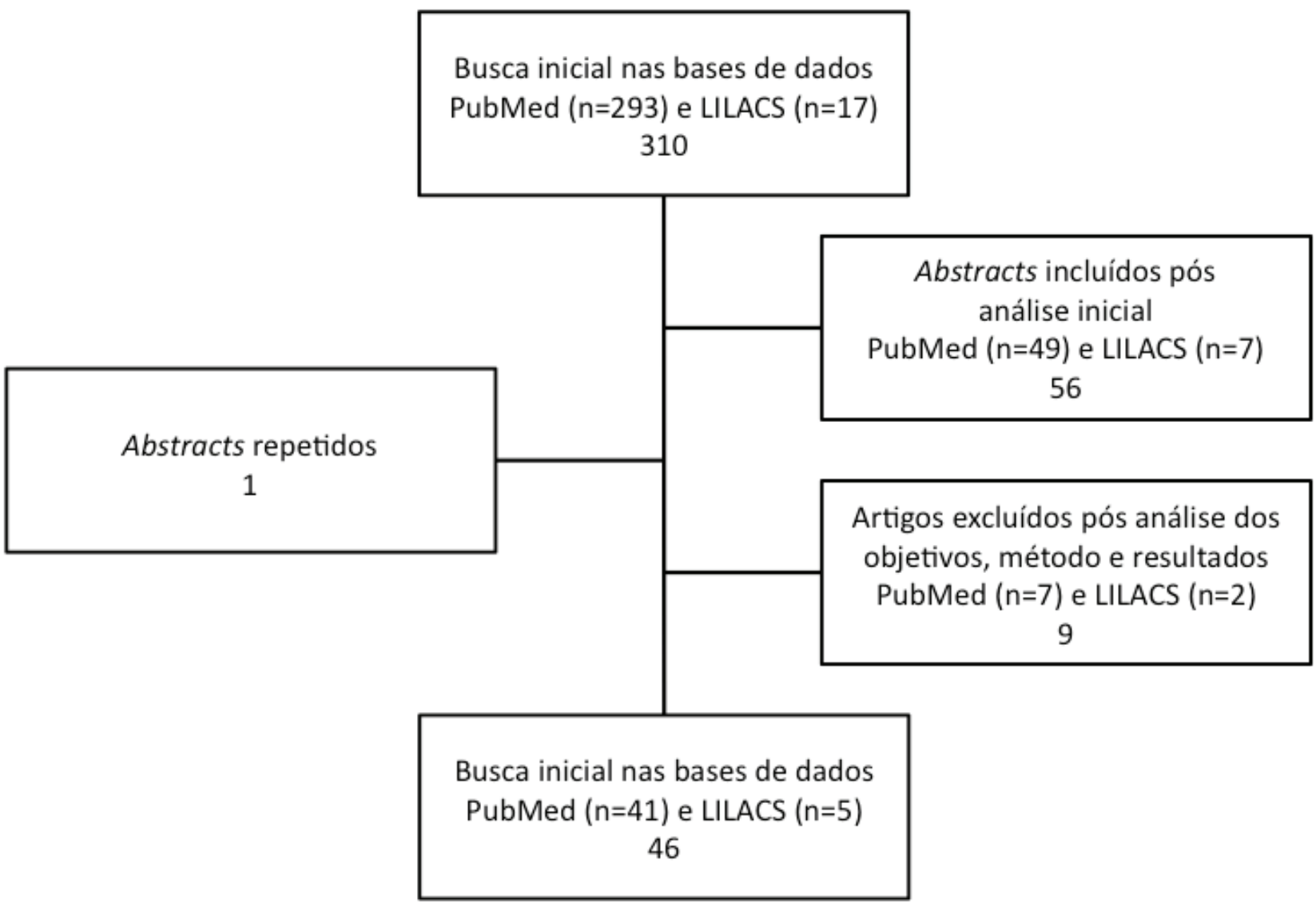

Figura 1 - Fluxo de análise de abstracts e artigos completos selecionados nas bases de dados PubMed e LILACS

Observa-se um número mais expressivo de artigos finais analisados na base de dados PubMed. Apenas um estudo estava repetido nas duas bases de dados, sendo, então, contabilizado apenas para a base LILACS. A Tabela 1 apresenta os instrumentos de avaliação da linguagem e seus subtestes, bem como a frequência de utilização de cada instrumento nos estudos dos artigos selecionados.

A Tabela 2 apresenta os instrumentos que avaliam um componente linguístico específico. Expõe, também, a frequência em que eles aparecem nos estudos.
A partir da Tabela 2 pode-se verificar que o Token Test e o Boston Naming Test são os instrumentos mais utilizados nas pesquisas analisadas. A Tabela 3 apresenta os instrumentos de avaliação da comunicação funcional e a descrição de cada um.

Os testes descritos na Tabela 3 apareceram, cada um, uma única vez, considerando os 46 artigos selecionados. Observa-se, ainda, a partir da comparação das Tabelas 1 e 3, que o uso de instrumentos de avaliação formal da linguagem foi mais expressivo que o de investigação funcional em pacientes com LHE. 
Tabela 1 - Baterias e instrumentos de avaliação de vários componentes linguísticos e respectivos subtestes

\begin{tabular}{|c|c|c|c|}
\hline Instrumento & Autores & Subtestes & $\begin{array}{c}\mathrm{N}^{\circ} \text { de } \\
\text { artigos (\%) }\end{array}$ \\
\hline $\begin{array}{l}\text { 1. Boston Diagnostic } \\
\text { Aphasia Examination } \\
\text { (BDAE) }\end{array}$ & $\begin{array}{l}\text { Mazaux e Orgogozo, 1982; Goodglass e } \\
\text { Kaplan, 1983; Kaplan, Goodglass e } \\
\text { Weintraub, 1983; Goodglass e Kaplan, } \\
\text { 1987; Goodglass, Kaplan, e Barresi, } \\
\text { 2001; Goodglass e Kaplan, 1972, 1983; } \\
\text { Goodglass e Kaplan, 1972. }\end{array}$ & $\begin{array}{l}\text { Discurso conversacional e } \\
\text { expositiva Compreensão } \\
\text { auditiva } \\
\text { Expressão oral } \\
\text { Leitura } \\
\text { Escrita } \\
\text { Praxias, } \\
\text { Visuocontrução } \\
\text { Agnosia } \\
\text { Cálculo } \\
\text { Orientação direita/esquerda }\end{array}$ & $17(36,96)$ \\
\hline $\begin{array}{l}\text { 2. Aachen Aphasia } \\
\text { Test (AAT) }\end{array}$ & $\begin{array}{l}\text { Huber, Poeck, Weniger e Willmes, 1983; } \\
\text { Graetz, De Bleser e Willmes, 1992; } \\
\text { Graetz, De Bleser e Willmes, 1991; } \\
\text { Luzzatti, Willmes, Bislacchi, De Blaser, } \\
\text { Faglia, Mazzocchi, Posteraro, Tarocco, } \\
\text { 1987; Luzzatti, Willmes, De Blaser, } \\
\text { Bianchi, Chiesa, De Tanti, Gonella, } \\
\text { Lorenzi, Bozzoli, 1994; Huber, Poeck, } \\
\text { Willmes, } 1984\end{array}$ & $\begin{array}{l}\text { Entrevista semi-estruturada } \\
\text { Token Test } \\
\text { Repetição } \\
\text { Escrita } \\
\text { Nomeação } \\
\text { Compreensão }\end{array}$ & $10(21,74)$ \\
\hline $\begin{array}{l}\text { 3. Psycholinguistic } \\
\text { Assessment of } \\
\text { Language Processing } \\
\text { in Aphasia (PALPA) }\end{array}$ & $\begin{array}{l}\text { Bastiaanse, Bosje e Visch-Brink, 1995; } \\
\text { Kay, Lesser e Coltheart, } 1996\end{array}$ & $\begin{array}{l}\text { Processamento linguístico } \\
\text { Processamento fonológico } \\
\text { (detecção de rimas, } \\
\text { segmentação e } \\
\text { discriminação auditiva) } \\
\text { Leitura } \\
\text { Escrita } \\
\text { Compreensão de frases, } \\
\text { palavras e imagens }\end{array}$ & $7(15,22)$ \\
\hline $\begin{array}{l}\text { 4. Western Aphasia } \\
\text { Battery (WAB) }\end{array}$ & Kertesz, 1982; Yiu, 1992 & $\begin{array}{l}\text { Fluência } \\
\text { Conteúdo de informação da } \\
\text { fala } \\
\text { Nomeação } \\
\text { Compreensão auditiva } \\
\text { Repetição } \\
\text { Leitura } \\
\text { Escrita } \\
\text { Testes não-verbais (desenho } \\
\text { do cubo, praxias, cálculo) }\end{array}$ & $6(13,04)$ \\
\hline $\begin{array}{l}\text { 5. Montreal-Toulouse } \\
\text { Battery }\end{array}$ & $\begin{array}{l}\text { Nespoulous, Lecours, Lafond, Joanette, } \\
\text { Lemay, Puel,Cot e Rascol, 1992; Béland } \\
\text { e Lecours, 1990; Nespoulous, Lecours, } \\
\text { Lafond, Lemay, Puel, Joanette, Cot e } \\
\text { Rascol, } 1986\end{array}$ & $\begin{array}{l}\text { Entrevista dirigida } \\
\text { Compreensão oral } \\
\text { Repetição } \\
\text { Linguagem automática } \\
\text { Compreensão oral de } \\
\text { palavras e frases } \\
\text { Leitura em voz alta } \\
\text { Compreensão escrita } \\
\text { Denominação } \\
\text { Praxias bucofaciais } \\
\text { Designação das partes do } \\
\text { corpo } \\
\text { Manipulação de objetos } \\
\text { Cópia } \\
\text { Leitura de texto } \\
\text { Ditado } \\
\text { Denominação escrita } \\
\text { Narração ora Compreensão } \\
\text { textual Narração escrita } \\
\text { Leitura de números } \\
\text { Repetição de números }\end{array}$ & $7(15,22)$ \\
\hline
\end{tabular}


Tabela 1 (continuação)

\begin{tabular}{|c|c|c|c|}
\hline Instrumento & Autores & Subtestes & $\begin{array}{c}\mathrm{N}^{\circ} \mathrm{de} \\
\text { artigos (\%) }\end{array}$ \\
\hline $\begin{array}{l}\text { 6. Lisbon Aphasia } \\
\text { Battery }\end{array}$ & Castro-Caldas, 1979 & $\begin{array}{l}\text { Fala espontânea Nomeação } \\
\text { visual Identificação de objeto } \\
\text { por nome } \\
\text { Compreensão de frase } \\
\text { Repetição de palavras e } \\
\text { frases } \\
\text { Span de dígitos } \\
\text { Leitura e escrita de palavras } \\
\text { Compreensão de texto e } \\
\text { escrita sob ditado. }\end{array}$ & $1(2,17)$ \\
\hline $\begin{array}{l}\text { 7. Batteria per l'Analisi } \\
\text { dei Deficit Afasici } \\
\text { (BADA) }\end{array}$ & Basso, Capitani, Zanobio, 1982 & $\begin{array}{l}\text { Discriminação auditiva e } \\
\text { visual } \\
\text { Repetição } \\
\text { Leitura } \\
\text { Escrita sob ditado Cópia } \\
\text { Decisão lexical (palavra, } \\
\text { não-palavra) Produção e } \\
\text { compreensão de frases } \\
\text { Noção de gramaticalidade } \\
\text { Reconhecimento e produção } \\
\text { de material verbal }\end{array}$ & $1(2,17)$ \\
\hline $\begin{array}{l}\text { 8. Standard } \\
\text { language test of } \\
\text { aphasia }\end{array}$ & Nirayama, 1975 & $\begin{array}{l}\text { Fala espontânea Nomeação } \\
\text { Compreensão } \\
\text { Leitura } \\
\text { Escrita, } \\
\text { Repetição e leitura de } \\
\text { fonemas }\end{array}$ & $1(2,38)$ \\
\hline $\begin{array}{l}\text { 9. Ullevaal Aphasia } \\
\text { Screening (UAS) }\end{array}$ & $\begin{array}{l}\text { Thommessen, Thoresen, Bautz-Holter, } \\
\text { Laake, } 1999\end{array}$ & $\begin{array}{l}\text { Expressão } \\
\text { Compreensão Repetição } \\
\text { Leitura } \\
\text { Repetição de uma sequência } \\
\text { de palavras } \\
\text { Escrita } \\
\text { Comunicação livre }\end{array}$ & $1(2,17)$ \\
\hline
\end{tabular}

Nota: A soma dos percentuais ultrapassa 100\%, na medida em que um mesmo estudo incluiu mais de um instrumento.

\section{REVISÃO DA LITERATURA}

A partir desta revisão de literatura que teve como objetivo verificar quais instrumentos de investigação de linguagem têm sido utilizados para avaliar quadros neurológicos súbitos de etiologia vascular envolvendo o $\mathrm{HE}$, observou-se que existe uma grande quantidade de baterias para este fim. Dentre as mais utilizadas internacionalmente estão: Boston Diagnostic Aphasia Examination (BDAE), Aachen Aphasia Test (AAT), Psycholinguistic Assessment of Language Processing in Aphasia (PALPA), Western Aphasia Battery (WAB) e Montreal-Toulouse Battery. Enquanto que nacionalmente destaca-se o uso do BDAE e da MontrealToulouse Battery ${ }^{25,30-33}$, contudo, ainda percebe-se à falta de instrumentos adaptados e padronizados para esta população ${ }^{19,24,29}$.

Os instrumentos de linguagem encontrados nesta pesquisa examinam, em sua maioria, os seguintes componentes linguísticos: discurso espontâneo, leitura, escrita, compreensão, nomeação e repetição, os quais podem estar prejudicados em maior ou menor grau em indivíduos com LHE ${ }^{4,5,18}$. A seguir serão descritos, brevemente, os cinco instrumentos mais utilizados encontrados nesta revisão, considerando as funções linguísticas avaliadas e as línguas em que foram adaptados e publicados.

O BDAE é a bateria de avaliação mais frequentemente aplicada em pesquisas internacionais ${ }^{7,34,35}$ e nacionais ${ }^{19,25,31,32}$, permite a verificação dos prejuízos que podem ocorrer pós LHE, como, de articulação, de fluência, as anomias, de repetição, de fala encadeada, de gramática, as parafasias, de compreensão auditiva, de leitura oral e compreensão leitora e de escrita e é capaz de classificar o tipo de afasia decorrente da lesão ${ }^{36}$. O BDAE já foi adaptado para diferentes línguas, como o francês e o espanhol, e apresenta diversas edições. As 
Tabela 2 - Instrumentos que avaliam componentes específicos de linguagem

\begin{tabular}{lllc}
\hline Instrumento & Autores & $\begin{array}{l}\text { Componente linguístico } \\
\text { avaliado }\end{array}$ & $\begin{array}{c}\mathbf{N}^{\circ} \text { de } \\
\text { artigos (\%) }\end{array}$ \\
\hline $\begin{array}{l}\text { 1. Boston Naming } \\
\text { Test }\end{array}$ & $\begin{array}{l}\text { Kaplan, Goodglass, Weintraub, 1978; } \\
\text { Kaplan, Goodglass, Weintraub, 1983; } \\
\text { Goodglass e Kaplan, 1983; } \\
\text { Goodglass, Kaplan e Barresi, 2001 }\end{array}$ & & $16(34,78)$ \\
\hline 2. Token Test & $\begin{array}{l}\text { De Renzi e Faglioni, 1978; De Renzi } \\
\text { e Vignolo, 1962; Huber et al., 1983; } \\
\text { De Renzi e Faglioni, 1968; McNeil, } \\
\text { Prescott, 1978 }\end{array}$ & Compreensão auditiva & $16(34,78)$ \\
\hline $\begin{array}{l}\text { 3. EC301 battery } \\
\text { 4. The John Hopkins } \\
\text { Dysgraphia Battery }\end{array}$ & Deloche, et al.,1995 & Habilidades aritméticas & $1(2,17)$ \\
\hline $\begin{array}{l}\text { 5. Birmingham } \\
\text { Object Recognition } \\
\text { Battery (BORB) }\end{array}$ & Riddoch e Humphreys, 1992 & Nomeação & $1(2,17)$ \\
\hline $\begin{array}{l}\text { 6. Peabody Picture } \\
\text { Vocabulary Test- }\end{array}$ & Dunn e Dunn, 1981 & & $1(2,17)$ \\
$\begin{array}{l}\text { Revised (PPVT-R, } \\
\text { Form M) }\end{array}$ & & Compreensão auditiva & $1(2,17)$ \\
\hline $\begin{array}{l}\text { 7. Cantonese } \\
\text { Linguistic } \\
\text { Communication } \\
\text { Measure (CLCM) }\end{array}$ & Kong, 2009 & & $1(2,17)$ \\
\hline $\begin{array}{l}\text { 8. Reading } \\
\text { Comprehension } \\
\text { Battery for Aphasia }\end{array}$ & LaPointe, Horner, 1979 & Discurso & \\
\hline $\begin{array}{l}\text { 9. Woodcock } \\
\text { Reading Mastery } \\
\text { Test-Revised }\end{array}$ & Woodcock, 1987 & & $1(2,17)$ \\
\hline
\end{tabular}

revisões realizadas desse instrumento ocorreram devido ao avanço das pesquisas neurolinguísticas incluindo métodos para avaliar complexidades narrativas e discursivas, dissociações específicas categóricas na compreensão/produção lexical, compreensão sintática e análise da conversão grafema/fonema durante a leitura ${ }^{18}$.

O AAT é um teste alemão, amplamente utilizado na Europa, traduzido e adaptado para o inglês, italiano, tailandês e holandês. É um instrumento capaz de diagnosticar, classificar e caracterizar quadros afásicos, tem sido largamente utilizado em pesquisas internacionais e é bastante similar ao BDAE, isto é, avalia domínios de linguagem semelhantes, tais como escrita e compreensão ${ }^{36-39}$.

O PALPA é uma avaliação psicolinguística do processamento da linguagem de pacientes afásicos. Avalia a compreensão e a expressão da fala e da escrita. Consiste de 60 testes rigorosamente controlados de componentes da estrutura da língua, como ortografia, fonologia, palavra e semântica da imagem, morfologia e sintaxe ${ }^{40}$. Foi adaptado para o espanhol, hebraico, holandês e português.

O WAB procura classificar os pacientes quanto ao tipo de afasia (Broca, Wernicke, global, mista e transcorticais), embora a classificação baseada nesse teste possa não ser consistente como as classificações de outras avaliações, como por exemplo, o BDAE. Os estudos encontrados nesta revisão aplicam este instrumento com o intuito de quantificar o grau de severidade e classificar a síndrome afásica, uma vez que este teste promove rápido olhar sobre o funcionamento do paciente ${ }^{36}$.

A Montreal-Toulouse Battery avalia diferentes componentes linguísticos e inclui também a avaliação das praxias verbais e não verbais, manipulação de objetos, reconhecimento de partes do corpo, orientação direita/esquerda e habilidades aritméticas. Nacionalmente, este instrumento é conhecido como Beta 86, o qual foi adaptado para o Português Brasileiro na década de 1980, não sendo padronizado para esta população. Entretanto, diversos estudos têm sido realizados com esta importante ferramenta clínica demonstrando sua 
Tabela 3 - Instrumentos de avaliação da comunicação funcional

\begin{tabular}{|c|c|c|}
\hline Instrumento & Autores & Descrição \\
\hline $\begin{array}{l}\text { 1. Lille Communication } \\
\text { Test }\end{array}$ & $\begin{array}{l}\text { Rousseaux, } \\
\text { Delacourt e } \\
\text { Wyrzykowski, } 2001\end{array}$ & $\begin{array}{l}\text { É composto por três partes: a) participação na } \\
\text { comunicação, b) comunicação verbal, } \\
\text { c) comunicação não verbal }\end{array}$ \\
\hline $\begin{array}{l}\text { 2. Functional } \\
\text { Communication Profile } \\
\text { (FCP) }\end{array}$ & Sarno, 1978 & $\begin{array}{l}\text { Entrevista com o paciente na qual são avaliados } \\
\text { comportamentos comunicativos comuns na vida } \\
\text { diária: a) movimento, b) uso de gestos, } \\
\text { c) compreensão, d) leitura, e) outros }\end{array}$ \\
\hline 3. Scenario test & $\begin{array}{l}\text { van der Meulen, } \\
\text { van de Sandt- } \\
\text { Koenderman e } \\
\text { Duivenvoordene } \\
\text { Ribbers, } 2010 \\
\end{array}$ & $\begin{array}{l}\text { Dramatização de seis situações, expostas em } \\
\text { cenas que retratam a vida diária. Permite a } \\
\text { comunicação verbal e não verbal; estratégias de } \\
\text { apoio à comunicação como: escrita, gestos, } \\
\text { desenhos, etc., além de um parceiro para auxílio. }\end{array}$ \\
\hline $\begin{array}{l}\text { 4. Communicative Abilities } \\
\text { in Daily Living (CADL) }\end{array}$ & Holland, 1980 & $\begin{array}{l}\text { Avalia habilidades comunicativas funcionais: } \\
\text { a) leitura, escrita e uso de números, b) atos de fala, } \\
\text { c) utilização do contexto, d) dramatização, } \\
\text { e) relações sequenciais, f) convenção social, } \\
\text { g) divergências, h) simbolismo não-verbal, } \\
\text { i) metáforas }\end{array}$ \\
\hline $\begin{array}{l}\text { 5. Communicative } \\
\text { Effectiveness Index (CETI) }\end{array}$ & $\begin{array}{l}\text { Lomas, Pickard e } \\
\text { Bester, } 1989\end{array}$ & $\begin{array}{l}\text { Avalia as seguintes situações comunicativas: } \\
\text { a) necessidades básicas, b) habilidades de vida, } \\
\text { c) necessidades sociais, e) cuidado com a saúde }\end{array}$ \\
\hline $\begin{array}{l}\text { 6. Amsterdam Nijmegen } \\
\text { Everyday Language Test } \\
\text { (ANELT) }\end{array}$ & $\begin{array}{l}\text { Blomert, Kean, } \\
\text { Koster e Schokker, } \\
1994\end{array}$ & $\begin{array}{l}\text { Avalia as habilidades comunicativas de pacientes } \\
\text { afásicos e estima as mudanças no decorrer do } \\
\text { tempo. }\end{array}$ \\
\hline
\end{tabular}

aplicabilidade 9,26,28,33,41. Atualmente, a MontrealToulouse Battery passou por uma nova adaptação e está sendo padronizada, normatizada e validada para o Português Brasileiro, tendo como base toda experiência adquirida por mais de 10 anos com estudos com o Beta $86^{42}$. Por sua importância, este instrumento também foi adaptado para a língua espanhola.

Apesar de haver inúmeras baterias para a avaliação da linguagem em indivíduos afásicos aparentemente completas, percebe-se ainda uma dificuldade no exame acurado de dissociações linguísticas nesses pacientes, ou seja, que possibilitem, com sensibilidade e especificidade, diagnosticar quais habilidades linguísticas preservadas e aquelas deficitárias para um trabalho em nível terciário de atenção a saúde mais preciso e eficaz. Tal dificuldade pode ser evidenciada pela necessidade de incluir mais de uma bateria em diferentes estudos para diagnosticar déficits de linguagem, como é o caso de uma pesquisa ${ }^{35}$ que para avaliar pacientes pós-AVC agudo utilizou o BDAE e a Montreal-Toulouse Battery, duas baterias extensas e com alguns subtestes semelhantes.

A partir da Tabela 3 observa-se que o Boston Naming Test e o Token Test avaliam nomeação e compreensão auditiva, respectivamente. São os instrumentos mais utilizados nas pesquisas, sendo, porém, comumente empregados como ferramentas complementares às baterias mais completas de avaliação linguística. Além disso, observa-se que grande parte dos instrumentos de linguagem encontrados nessa revisão possuem subtestes de nomeação. Esse achado deve estar relacionado à alta incidência de quadros de anomia pós LHE, bem como ao elevado grau de disfunção e prejuízo na qualidade de vida destes pacientes quando se tornam anômicos ${ }^{43}$.

Observa-se também a utilização de instrumentos de comunicação funcional para avaliar indivíduos afásicos, uma vez que tal população pode apresentar prejuízos lexicais, sintáticos e pragmáticos severos ${ }^{4,44,45}$. Os déficits pragmáticos são observados principalmente, quando novas informações são apresentadas, um novo tópico é introduzido, um discurso é organizado logicamente e na adaptação do conhecimento ao interlocutor, podendo prejudicar em algum nível a independência funcional comunicativa no cotidiano ${ }^{4}$.

Os instrumentos de avaliação de linguagem, sejam formais ou funcionais, além de permitirem um diagnóstico mais preciso, objetivo e quantitativo, podem servir como estratégia de tratamento. Isto porque subtestes delineados primeiramente 
para detectar prejuízos podem embasar o desenvolvimento de tarefas linguísticas similares, com o objetivo de tratar e/ou de acompanhar a evolução do caso.

Dessa forma, a partir desta revisão, foi possível constatar que todos os instrumentos utilizados, internacionalmente, apresentam adaptações e padronizações apropriadas para a realidade social, cultural e linguística da população avaliada. No contexto nacional, são escassas as ferramentas clínicas padronizadas para o Português Brasileiro dedicadas exclusivamente à linguagem. Tal lacuna pode estar relacionada à dificuldade de se padronizar um teste para o contexto nacional, uma vez que envolve culturas bastante diversas e para isso necessita, além de apoio financeiro, parcerias entre instituições para padronização multicêntrica ${ }^{29}$.

No entanto, muitos estudos nacionais 9,19,20,27,46 têm abordado a avaliação da linguagem com intuito de analisar o desempenho de populações saudáveis, o que contribui para a análise de resultados de pacientes clínicos. Porém, ainda há poucos estudos nacionais que avaliam pacientes afásicos ${ }^{25,31-33}$ em comparação com estudos internacionais.

Devido à demanda clínica de diagnóstico, prognóstico e plano de reabilitação cada vez maior para auxiliar indivíduos acometidos por lesões cerebrais súbitas, se faz necessária a construção e/ou adaptação de instrumentos mais específicos de avaliação da linguagem para a população brasileira. Tais ferramentas precisam abranger componentes linguísticos condizentes com as funções comunicativas do $\mathrm{HE}$, como tarefas que examinem discurso, pragmática, compreensão oral e escrita de palavras, frases e textos, nomeação de figuras simples e complexas, cálculo, praxias, orientação, repetição, designação de parte do corpo, manipulação de objetos, fluências verbais, fala espontânea, linguagem automática e leitura. Isto é, instrumentos que envolvam diferentes componentes da linguagem, com diferentes modalidades de input e de output para uma análise acurada do perfil linguístico dos diferentes pacientes.

Destaca-se, também, a escassez de estudos encontrados com ferramentas de avaliação breve, apontando para uma importante e restritiva lacuna ${ }^{23}$. A necessidade desses instrumentos é indubitável, tendo em vista que no contexto ambulatorial o uso de ferramentas com poder de auxílio diagnóstico em tempo breve parece ser uma opção adequada para a investigação de diversos componentes linguísticos, pois demanda menos tempo podendo oferecer uma melhor orientação para uma avaliação posterior mais extensa e aprofundada para determinadas funções e diferentes quadros neurológicos.
Apesar da identificação de diferentes baterias de linguagem nesta revisão, percebe-se uma lacuna de alguns instrumentos importantes que não foram encontrados para este estudo e que são consagrados na literatura. Destacam-se dentre estas ferramentas o MAE e o BASA, por exemplo, e o Token Test reduzido, recentemente adaptado e normatizado para a população brasileira ${ }^{47}$. Entretanto, realizou-se uma extensa busca a partir de um levantamento de palavras-chaves utilizadas em livros e artigos que abordavam avaliações da linguagem em adultos com lesão cerebral com o intuito de abranger o maior número de dados relevantes. Mesmo oferecendo um panorama sobre avaliação das afasias o método de revisão sistemática apresenta uma limitação inerente a partir das bases indexadoras e dos termos utilizados, podendo não incluir alguns estudos encontrados por outros métodos de busca.

\section{CONCLUSÃO}

A partir dessa revisão sistemática foi possível identificar nove instrumentos utilizados internacionalmente e duas nacionais, que avaliam componentes linguísticos em pacientes com AVC de $\mathrm{HE}$. Embora haja esta quantidade de ferramentas disponibilizadas, em contexto nacional observa-se que existem estudos que avaliam componentes da linguagem em populações saudáveis, porém ainda são escassos os estudos com estes instrumentos envolvendo especificamente pacientes com LHE. Assim, atenta-se para o fato de que a linha de pesquisa com etapas de adaptação e/ou construção de instrumentos que visem ao exame de componentes linguísticos em adultos ainda é incipiente.

A avaliação quantitativa e qualitativa da linguagem torna-se essencial nos quadros envolvendo o $\mathrm{HE}$, pois ajuda no diagnóstico e principalmente no delineamento terapêutico, além de possibilitar a compreensão de como outros processos cognitivos podem encontrar-se alterados devido a déficits linguísticos. Isto porque diversas funções, como a memória, tendem a ser diretamente influenciadas pela linguagem. Além disso, alterações na expressão e compreensão da linguagem, oral e escrita, podem afetar drasticamente a relação do paciente com seu ambiente social, ocupacional e familiar. Dessa forma, são necessários esforços para o desenvolvimento e normatização de instrumentos de linguagem para avaliação de pacientes adultos com LHE e outros quadros neurológicos adequados à realidade sociocultural brasileira. 


\begin{abstract}
The theme of this study is the language assessment in patients with left brain damage (LBD). It is necessary as it helps to establish the diagnosis, prognosis and rehabilitation planning, adding findings to clinical observation, interview and tasks. The purpose is to identify which language instruments have been used to assess non-progressive neurological disorders involving the left hemisphere (LH) and to analyze which language components are the most assessed ones. It was possible to identify nine international instruments that assess different components of language in LBD patients. In the national context, however, only two batteries to assess this population were found, highlighting the need for construction and/or adaptation of language assessment tools for the Brazilian population. Naming and oral comprehension were the most examined linguistic components, respectively. The evaluation of language is essential in the neurological disorders that affect the $\mathrm{LH}$, because language deficits can lead to an impaired social, occupational and family life.
\end{abstract}

KEYWORDS: Evaluation; Stroke; Language Tests

\section{REFERÊNCIAS}

1. Van Lancker DV. Rags to riches: our increasing appreciation of cognitive and communicative abilities of the human right cerebral hemisphere. Brain Lang. 1997; 57:1-11.

2. Joanette $Y$, Goulet $P$, Hannequin D. Right hemisphere and verbal communication. New York: Springer; 1990.

3. Talarico TR, Venegas MJ, Ortiz KZ. Perfil populacional de pacientes com distúrbios da comunicação humana decorrentes de lesão cerebral, assistidos em hospital terciário. Rev CEFAC. 2011; 13(2):330-9.

4. Rousseaux M, Daveluy W, Kozlowsk O. Communication in conversation in stroke patients. $\mathrm{J}$ Neurol. 2010; 257:1099-107.

5. Pedersen PM, Vinter K, Olsen TS. Aphasia after stroke: type, severity and prognosis. The Copenhagen aphasia study. Cerebrovasc Dis. 2004; 17:35-43.

6. Donovan NJ, Kendall DL, Heaton SC, Kwon S, Velozo CA, Duncan PW. Conceptualizing Functional Cognition in Stroke. Neurorehabil Neural Repair. 2008; 22: 122-35.

7. D'Arcy RCND, Marchand Y, Eskes GA, Harrison ER, Phillips SJ, Major A et al. Electrophysiological assessment of language function following stroke. Clin Neurophys. 2003; 114: 662-72.

8. Kalbe E, Reinhald N, Brand M, Marowitsch $\mathrm{H}$, Kessler J. A new test battery to assess aphasic disturbances and associated cognitive dysfunctions. German Normative data on the aphasia check list. J Clin Exp Neuropsy. 2005; 24: 779-94.

9. Soares ECS, Ortiz KZ. Influence of brain lesion and educational background on language tests in aphasic subjects. Demen Neuropsychol. 2008; 2: 321-7.

10. Helm-Estabrooks N. Aphasia Diagnostic Profiles (ADP). Dallas: PRO-ED; 1992.

11. Nakase-Thompson R, Manning E, Sherer M, Yablon SA, Gontkovsky SLT, Vickery C. Brief assessment of severe language impairments: Initial validation of the Mississippi aphasia screening test. Brain Injury. 2005; 19(9): 685-91.

12. Goodglass H, Kaplan E. Assessment of aphasia and related disorders. Philadelphia: Lea \& Febiger; 1972.

13. Frattali CM, Thompson C, Holland A, Wohl A, Ferketic $M$. American-Speech-LanguageHearing Association Functional Assessment of Communication Skills for Adults. Rockville, MD: American Speech-Language-Hearing Association, 1995.

14. Benton L, Hamsher S, Sivan A. Multilingual Aphasia Examination (MAE-E). Journal of the International Neuropsychological Society. Cambridge University Press; 1994.

15. Porch B. Porch Index of Comunicative Ability (PICA). Palo Alto: Consulting Psychologists Press; 1981.

16. Hilari K, Northcott S, Roy P, Marshall J, Wiggins RD, Chataway J, Ames D. Psychological distress after stroke and aphasia: the first six months. Clin Rehabil. 2010; 24:181-90.

17. Kertesz A. Western Aphasia Battery. New York: Grune \& Stratton; 1982.

18. Goodglass H, Kaplan E, Barresi B. The Assessment of Aphasia and Related Disorders. $3^{\text {a }}$ ed. Philadelphia, PA: Lippincott, Williams and Wilkins; 2001. 
19. Radanovic M, Mansur LL. Performance of a Brazilian population sample in the Boston Diagnostic Aphasia Examination. A pilot study. Braz J Med Biol Res. 2002; 35(3): 305-17.

20. Radanovic M, Mansur LL, Scaff M. Normative data for the Brazilian population in the Boston Diagnostic Aphasia Examination: influence of schooling. Braz J Med Biol Res. 2004; 37(11): 1731-8.

21. Joanette, Y., Goulet, P., \& Hannequin D. Right hemisphere and verbal communication. New York: Springer-Verlag; 1990.

22. Fonseca RP, Parente MAMP, Côte H, Ska $B$, Joanette $Y$. Apresentando um instrumento de avaliação da comunicação à Fonoaudiologia Brasileira: Bateria MAC. Rev Pró-fono. 2008; 285-91.

23. Casarin FS, Pagliarin KC, Koehler C, Oliveira $\mathrm{CR}$, Fonseca RP. Instrumentos de avaliação breve da comunicação: ferramentas existentes e sua aplicabilidade clinica. Rev CEFAC. 2011; 13(5): 917-25.

24. Fonseca RP, Parente MAMP, Côte H, Ska B, Joanette Y. Bateria MAC - Bateria Montreal de Avaliação da Comunicação. São Paulo: Pró-Fono; 2008.

25. Mansur LL, Radanovic M, Taquemori L, Greco L, Araújo GC. A study of the abilities in oral language comprehension of the Boston Diagnostic Aphasia Examination - Portuguese version: a reference guide for the Brazilian population.Braz $\mathrm{J}$ Med Biol Res. 2005; 38(2):277-92.

26. Soares ECS, Ortiz KZ. Influence of schooling on language abilities of adults without linguistic disorders. Sao Paulo Med J. 2009; 127(3):134-9.

27. Ortiz KZ, Costa FP. M1-Alpha test in normal subjects with low educational level: a pilot study.J Soc Bras Fonoaudiol. 2011; 23(3):220-6.

28. Ortiz KZ, Ferreira CP, Bento ACP. Aplicação do Teste Beta 86(protocolo MT modificado) em analfabetos. Fono Atual. 2006; 35: 65-73.

29. Giusti E, Befi-Lopes DM. Tradução e adaptação transcultural de instrumentos estrangeiros para o Português Brasileiro (PB). Rev Pro-Fono. 2008; 20(3): 207-10.

30. Ortiz KZ, Osborn E, Chiari BM. O teste M1Alpha como instrumento de avaliação da afasia. Rev Pro-Fono. 1993; 23-9.

31. Radanovic M, Mansur LL, Azambuja MJ, Porto CS, Scaff M. Contribution to the evaluation of language disturbances in subcortical lesions. Arq Neuropsiquiatr. 2004; 62(1):51-7.

32. Mansur, Radanovic M, Ruegg D, Mendonça LIZ, Scaff M. Descriptive study of 192 adults with speech and language disturbances. Sao Paulo Med J/Rev Paul Med. 2002; 120(6):170-4.
33. Santos AD, Ortiz KZ. Comparação do desempenho de pacientes afásicos em diferentes testes de compreensão oral. Fon Atual. 2005, 33(8): 26-32.

34. Naeser MA, Martin PI, Lundgren K, Klein R, Kaplan J, Treglia E et al. Improved Language in a Chronic Nonfluent Aphasia Patient After Treatment With CPAP and TMS. Cog Behav Neurol. 2010; 23: 29-38.

35. Dewarrat GM, Anonni J-M, Fornari E, Carota A, Bogousslavsky J, Maeder P. Acute aphasia after right hemisphere stroke. J Neurol. 2009; 256:1461-7.

36. Hegde MN, Freed D. Assessment of communication disorders in adults. San Diego: Plural Publishing; 2011.

37. Huber W, Poeck K, Willmes K. The Aachen Aphasia Test. Adv Neurol.1984; 42:291-303.

38. Code C, Torney A, Gildea E, Willmes K. Outcome of a One-Month Therapy Intensive for Chronic Aphasia: Variable Individual Responses. Semin Speech Lang. 2010; 31(1): 21-33.

39. Szaflarski JP, Ball AL, Grether S, Al-fwaress F, Griffith NM, Neils-Strunjas J, Newmeyer A, Reichhardt R. Constraint-induced aphasia therapy stimulates language recovery in patients with chronic aphasia after ischemic stroke. Med Sci Monit. 2008; 14(5): CR243-CR50.

40. Kay J, Lesser R, Coltheart M. Psycholinguistic Assessment of Language Processing in Aphasia (PALPA): an introduction. Aphasiology. 1996; 10(2): 159-215.

41. Lecours AR, Parente MAMP, Feijó AV, Maia A. Anartria pura: estudo de dois casos. Psico Reflexao Crítica. 2001; 14:367-77.

42. Parente MAMP, Ortiz KZ, Soares-Ishigaki E, Fonseca RP, Joanette Y, Lecours AR, Nespoulous J-L. Bateria Montreal Toulouse de Avaliação da Linguagem (MTL-Brasil). São Paulo: Vetor. no prelo.

43. Fridriksson J, Baker JM, Moser D. Cortical mapping of naming errors in aphasia. Hum Brain Mapp. 2009;30(8):2487-98.

44. Carlomagno S, Pandolf M, Labruna L, Colombo A, Razzano C. Recovery From Moderate Aphasia in the First Year Poststroke: Effect of Type of Therapy. Arch Phys Med Rehabil. 2001; 82: 1073-80.

45. van der Meulen I, van de Sandt-Koenderman WM, Duivenvoordene HJ, Ribbers GM. Measuring verbal and non-verbal communication in aphasia: reliability, validity, and sensitivity to change of the Scenario Test. Int J Lang Commun Dis. 2010; 45: 424-35. 
46. Machado O, Correia SM, MansurLL. Performance of normal Brazilian adults in a semantic test: effect of literacy. Pró-Fono. 2007;19 (3): 289-94.

47. Moreira L, Chlottfeld CG, de Paula JJ, Daniel MT, Paiva A, Cazita V, Coutinho G, Salgado JV,
Malloy-Diniz LF. Estudo Normativo do Token Test versão reduzida: dados preliminares para uma população de idosos brasileiros. Rev psiquiatr clín. 2011; 38 (3): 97-101.

http://dx.doi.org/10.1590/S1516-18462013005000015

RECEBIDO EM: 22/09/2011

ACEITO EM: 29/01/2012

Endereço para correpondência :

Karina Carlesso Pagliarin

PUCRS - Avenida Ipiranga, 6681, prédio11 -

9 o andar, sala 932

Porto Alegre - RS

CEP: 90619-900

E-mail: karinap_fono@yahoo.com.br

Rev. CEFAC. 2013 Mar-Abr; 15(2):444-454 